\title{
La alfabetización en salud como factor clave en el autocuidado de la dieta en personas con diabetes mellitus tipo 2
}

\author{
Health literacy as a key factor in diet self-care in patients with type 2 diabetes mellitus \\ Edgar Humberto León-Landa' ${ }^{1}$ orcid.org/0000-0002-2069-6792 \\ Liliana Orozco-Castillo² orcid.org/0000-0002-8686-6168 \\ Vianey Guadalupe Argüelles-Nava' orcid.org/0000-0002-5878-2522 \\ Lucía Hernández-Barrera3 ${ }^{3}$ orcid.org/0000-0002-0963-5710 \\ Manuel Salvador Luzanía-Valerio ${ }^{1}$ orcid.org/0000-0002-9377-6291 \\ Yolanda Campos-Uscanga ${ }^{*}$ orcid.org/0000-0002-5114-3621
}

\begin{abstract}
Instituto de Salud Pública, Universidad Veracruzana. Veracruz, México
Universidad de Caldas. Manizales, Colombia

Instituto Nacional de Salud pública. Morelos, México
\end{abstract}

\section{Resumen}

Introducción: Alfabetización en Salud (AS) es un término que proviene del inglés health literacy, se refiere al conjunto de habilidades sociales y cognitivas que determinan la capacidad de una persona para acceder, entender y utilizar la información de forma que le permita mantener un buen estado de salud. Objetivo: Determinar la asociación entre AS y las conductas de autocuidado en personas con diabetes mellitus tipo 2. Materiales y métodos: Estudio cuantitativo, transversal y analítico llevado a cabo en dos centros de salud de atención primaria de la ciudad de Xalapa, Veracruz, México entre agosto-noviembre de 2016. La AS se midió con el European Health Literacy Survey (HLS-EU-Q47) y las conductas de autocuidado con el Summary of Diabetes Self-care Activities (SDSCA) de Toobert y Glasgow. Se eligieron 90 personas con diabetes mellitus tipo 2 que acudían a consulta. La participación fue voluntaria y previa firma de consentimiento informado. Resultados: El autocuidado de la dieta mostró asociación con la AS en su dimensión de promoción de la salud $(\mathrm{p}=0,018)$. Conclusiones: La AS se relaciona con las conductas de autocuidado, en específico de la dieta; a medida que aumenta la AS mejora el autocuidado.

Palabras clave: Alfabetización en salud; diabetes mellitus tipo 2; autocuidado; educación en salud. (Fuente: DeCS, Bireme).

\begin{abstract}
Introduction: Health literacy refers to the set of social and cognitive skills that define the person's ability to access, understand and utilize information in such a way that a healthy condition can be maintained. Objective: To determine the association between AS and self-care behaviors in patients with type 2 diabetes mellitus. Materials and methods: A quantitative, cross-sectional and analytical study carried out in two primary health care centers in the city of Xalapa, Veracruz-Mexico, from August to November 2016. The AS was measured through the European Health Literacy Survey (HLSEU-Q47) and the self-care behaviors were assessed using the Summary of Diabetes Self-care Activities (SDSCA) by Toobert and Glasgow. We chose 90 people with type 2 diabetes mellitus who attended consultation at the health care centers. Although their participation was voluntary, participants were required to sign the informed consent form. Results: Diet selfcare was associated with AS, in a health promotion context $(\mathrm{p}=0.018)$. Conclusions: AS is directly related to self-care behaviors, specifically to diet.
\end{abstract}

Key words: Health literacy; diabetes mellitus, type 2; self-care, health education. (Source: DeCS, Bireme). 


\section{Introducción}

Alfabetización en salud (AS) se refiere a "las habilidades sociales y cognitivas que determinan el nivel de motivación y la capacidad de una persona para acceder, entender y utilizar la información de forma que le permita promover y mantener una buena salud"(1). La AS es parte de las actividades de educación en salud, va más allá de transmitir información y desarrollar habilidades para poder leer folletos y agendar citas médicas con éxito(2). Se trata de mejorar el acceso de las personas a la información en salud y su capacidad para usarla efectivamente, es decir, apoyar el proceso de empoderamiento y propiciar conductas de autocuidado para el control de su salud. Se ha evidenciado que el nivel bajo de AS constituye un problema de salud pública, ya que se asocia con escaso conocimiento en términos relacionados a la salud, mayor uso de los servicios de salud públicos $\mathrm{y}$ menores conductas de autocuidado(3).

Por otra parte, la atención médica de la diabetes absorbe $12 \%$ del gasto global en salud a nivel mundial y su tasa de mortalidad es muy elevada, en el año 2014 murieron 5 millones de personas a causa de esta enfermedad o por alguna complicación asociada con ella(4). En México uno de los cinco principales motivos de consulta de personas en edad de 20 a 49 años es la diabetes, y es la principal causa de consulta en personas de 50 años o más, se sitúa también como el motivo más frecuente de hospitalización en hombres y mujeres ${ }^{(3)}$.

De acuerdo con la Encuesta Nacional de Salud y Nutrición (ENSANUT) del año 2016, del total de la población adulta en México 9,4\% refirió tener un diagnóstico médico de diabetes, de ellos 87,8\% reciben tratamiento para su control, no obstante, solo el 24,5\% reportan un control adecuado, es decir tres de cada cuatro pacientes con diabetes no tienen control del padecimiento(5).

La principal recomendación para el control de la diabetes en los pacientes es el cambio de estilo de vida incluyendo actividad física regular, suficientes horas de sueño, dejar de fumar y reducción gradual del exceso de peso mediante una dieta balanceada prescrita por el profesional en nutrición(6).

Si bien el tratamiento de la enfermedad debe ser multidisciplinar y conlleva un compromiso de autocuidado de la persona con diabetes, este es escasamente promovido en las instituciones de salud, ya que se da por hecho que es una capacidad inherente del paciente. Aunado a esto se tiende a medicalizar la atención, descuidando los componentes nutricional, de actividad física, sueño, entre otros $(7,8)$.

De acuerdo con Dorotea Orem entre los requisitos de autocuidado ante la desviación de salud, se encuentran la búsqueda y aseguramiento de asistencia médica, atender los efectos de la condición patológica $\mathrm{y}$ las prescripciones hechas por profesionales de la salud(9). El autocuidado como respuesta a indicaciones médicas incluye acciones que se realizan en la vida cotidiana como la observación de síntomas, el seguimiento y ajuste de las indicaciones médicas, la búsqueda de información en torno al malestar, ajustes en hábitos de alimentación y actividad física que resultan clave para el control de la enfermedad(10).

En investigaciones recientes se ha encontrado una relación entre bajo nivel de AS y menor autoeficacia en el cuidado y control de la diabetes(11-13). De igual manera, entre las conductas de autocuidado y el nivel de conocimiento de los pacientes con diabetes, así como una influencia del autocuidado en el estado de salud(14-16).

En México, la mayoría de los estudios que buscan identificar los factores relacionados con el autocuidado en pacientes con diabetes se han enfocado en el apoyo social, el grado de analfabetismo y las creencias populares $(10,17)$, sin embargo, no existen estudios que analicen el nivel de alfabetización y cómo puede afectar en la comprensión de los mensajes en salud (indicaciones médicas, información sobre la enfermedad, metas del tratamiento, agenda de citas, etcétera) y en consecuencia, en las conductas de autocuidado; dando origen a su ausencia en los programas de salud pública enfocados en la prevención y tratamiento de la diabetes; por lo cual el objetivo de este trabajo es determinar la asociación entre la AS y las conductas de autocuidado en personas con diabetes tipo 2 .

\section{Materiales y métodos}

\section{Participantes}

Se realizó un estudio cuantitativo, de diseño transversal y analítico en dos centros de salud de la 
ciudad de Xalapa, Veracruz, México en las salas de espera durante los meses de agosto a noviembre de 2016. Los criterios de inclusión consideraron a personas mayores de 18 años, con diagnóstico de diabetes mellitus tipo 2, que asistían a consulta médica de control. El tamaño muestral estimado fue de 86 personas, considerando una correlación a detectar de 0,297 $(\mathrm{p}<0,01)$ entre comportamientos de autocuidado y alfabetización en salud documentada en un estudio previo(11). El nivel de confianza utilizado para el cálculo fue de 95\%, con una potencia estadística del $80 \%$.

\section{Instrumentos}

Para evaluar la AS se utilizó la versión en español del European Health Literacy Survey (HLS-EU-Q47)(18), que consta de 47 ítems que permiten identificar 3 dimensiones: atención y cuidado sanitario; prevención de enfermedades; y promoción de la salud, mismas que a su vez están integradas por cuatro capacidades: búsqueda y acceso a la información, comprensión, saber evaluar y juzgar dicha información y, por último, saber aplicarla(18,19).

El instrumento permite clasificar en cuatro niveles de alfabetización en salud: inadecuada (0-25), problemática (>25-33), suficiente (>33-42) y excelente (>42-50). Para calcular el índice de alfabetización en salud se atribuyeron los siguientes valores a las respuestas: 1 "muy difícil", 2 "difícil", 3" fácil", 4 "muy fácil"; se calculó la media de cada participante y se utilizó la siguiente formula: índice = (media-1) * (50/3)(18). En la muestra del presente estudio el instrumento mostró una confiabilidad alta en la escala global $(\alpha=0,91)$ y aceptable para las dimensiones: atención y cuidado sanitario $(\alpha=0,67)$, prevención de enfermedades $(\alpha=0,79)$, promoción de la salud $(\alpha=0,71)$, acceder $(\alpha=0,83)$, entender $(\alpha$ $=0,55)$, evaluar $(\alpha=0,66)$ y aplicar $(\alpha=0,64)$.

El autocuidado se midió con la versión en español del Sumary of Diabetes Self-care Activities (SDSCA) desarrollado por Toobert, Hampson y Glasgow, el instrumento mide las conductas de autocuidado que ha tenido el paciente en los últimos 7 días. Para esta investigación se utilizó la versión de 12 ítems traducida al español y validado por Vincent, Pasvogel y Barrera en el año 2007(20).

Para las variables sociodemográficas se utilizó un cuestionario sobre datos personales y se obtuvo de la historia clínica del participante los datos de peso, talla, IMC, glucemia y complicaciones de la diabetes. Para determinar el control glucémico de los participantes se empleó el nivel de glucemia tomado el día de la última consulta y se clasificó de acuerdo a la Norma Oficial Mexicana 015 que identifica a un paciente en control cuando presenta glucemia en ayuno entre 70 y $130 \mathrm{mg} / \mathrm{dl}$ y paciente sin control cuando la glucemia en ayuno es $>130 \mathrm{mg} / \mathrm{dl})^{(8)}$.

\section{Análisis de datos}

Se analizaron los datos con el software estadístico "Statistical Package for the Social Sciences" (SPSS) versión 23 , se describieron las variables cuantitativas (edad, alfabetización en salud y autocuidado) a través de media y desviación estándar y las cualitativas (sexo, nivel de escolaridad, estado civil y complicaciones de la diabetes) con frecuencias y proporciones. Se realizaron análisis de varianza (ANOVA) de todas las dimensiones de la alfabetización en salud entre grupos según el nivel de escolaridad (sin estudios, primaria, secundaria y bachillerato o más) y edad de los encuestados (50 años o menos, 51 a 65 y mayores de 65), la prueba de Levene se utilizó para determinar la igualdad de varianzas. Para identificar asociaciones entre variables, se realizó análisis del coeficiente de correlación de Pearson y regresión lineal para las variables predictoras de autocuidado de la dieta, incluyendo las dimensiones y capacidades de la alfabetización en salud y la edad. En los modelos multivariados se incluyeron variables que seguían una distribución normal y que cumplieron con los demás supuestos. Para todas las pruebas se consideró significativo un valor de $p$ inferior a 0,05.

\section{Aspectos éticos}

El protocolo de investigación fue evaluado y registrado por el Comité de Ética e Investigación de los Servicios de Salud de Veracruz, asignando el folio SEIC-007-2016. La participación fue voluntaria y previa firma del consentimiento informado, los datos recabados solo fueron utilizados para fines de la investigación y se guardó anonimato.

\section{Resultados}

La mayoría de los participantes en el estudio fueron mujeres $(72,2 \%)$, con una edad promedio de 59 años $( \pm 11,3)$, la mayoría refirió estar casado $(45,6 \%)$, mientras que cerca del $40 \%$ se encuentra sin algún tipo de relación de pareja (Tabla 1). 
Tabla 1. Descripción general de la muestra

\begin{tabular}{|c|c|}
\hline Variable & $n=90$ \\
\hline \multicolumn{2}{|l|}{ Sexo } \\
\hline Hombre, n (\%) & $25(27,8)$ \\
\hline Mujer, n (\%) & $65(72,2)$ \\
\hline Edad (años), media \pm DE & $59,4 \pm 11,3$ \\
\hline \multicolumn{2}{|l|}{ Escolaridad, n (\%) } \\
\hline Sin estudios & $27(30)$ \\
\hline Primaria & $25(27,8)$ \\
\hline Secundaria & $25(27,8)$ \\
\hline Bachillerato o más & $13(14,4)$ \\
\hline \multicolumn{2}{|l|}{ Índice de Masa Corporal, n (\%) } \\
\hline Normal & $21(23,3)$ \\
\hline Sobrepeso & $36(40,0)$ \\
\hline Obesidad & $33(36,7)$ \\
\hline Media de IMC $\left(\mathrm{kg} / \mathrm{m}^{2}\right) \pm \mathrm{DE}$ & $28,5+4,8$ \\
\hline En control glucémico* & $35(38,9)$ \\
\hline $\begin{array}{l}\text { Tiempo de duración de la diabetes (años), } \\
\text { media } \pm \text { DE }\end{array}$ & $9,6 \pm 7,6$ \\
\hline Sigue algún plan de alimentación, n (\%) & $65(72,2)$ \\
\hline \multicolumn{2}{|l|}{ Conductas de autocuidado, media \pm DE } \\
\hline Autocuidado de la dieta & $14,7 \pm 5,9$ \\
\hline Autocuidado del ejercicio & $3,2 \pm 3,9$ \\
\hline Automonitoreo de la glucosa & $1,3 \pm 1,4$ \\
\hline Toma medicamento & $6,7 \pm 1,0$ \\
\hline \multicolumn{2}{|l|}{ Complicaciones propias de la diabetes, $n(\%)$} \\
\hline Retinopatía diabética & $33(36,7)$ \\
\hline Pie diabético & $11(12,2)$ \\
\hline Nefropatía & $12(13,3)$ \\
\hline Neuropatía & $28(31,1)$ \\
\hline Enfermedades cardiovasculares & $1(1,1)$ \\
\hline
\end{tabular}

El $38,9 \%$ de la muestra se encontraba en control glucémico. En cuanto al tiempo de duración de la diabetes, la mayoría refirió tener 10 años o menos desde el diagnóstico. El 45,6\% tuvo un inadecuado nivel de AS, y sólo el 6,7\% alcanzó el nivel suficiente (Tabla 2).

Tabla 2. Descripción de la alfabetización en salud en la muestra

\begin{tabular}{lr}
\hline Variable & $\mathbf{n = 9 0}$ \\
\hline Dimensiones y capacidades (media \pm DE) & \\
Atención y cuidado & $44,86 \pm 5,7$ \\
Prevención de enfermedades & $39,94 \pm 4,4$ \\
Promoción de la salud & $33,50 \pm 6,1$ \\
Acceder/ obtener & $31,24 \pm 4,7$ \\
Entender/comprender & $30,25 \pm 4,1$ \\
Procesar/juzgar & $30,39 \pm 3,9$ \\
Aplicar/usar & $26,77 \pm 3,6$ \\
Índice de alfabetización en salud, n (\%) & \\
Inadecuado & $41(45,6)$ \\
Problemático & $43(47,8)$ \\
Suficiente & $6(6,7)$ \\
\hline DE= Desviación estándar &
\end{tabular}

Se observaron diferencias significativas por nivel de escolaridad, en las medias de todas las dimensiones de alfabetización en salud (Tabla 3). El nivel de AS en las dimensiones de atención y cuidado, y prevención de enfermedades, es mayor conforme aumenta el nivel de estudios (Figura 1).

Tabla 3. Comparación de medias de dimensiones y capacidades de alfabetización en salud de acuerdo con el nivel de estudios

\begin{tabular}{llrrrr}
\hline \multicolumn{1}{c}{ Dimensión } & & Media & DE & F & P \\
\hline Atención y cuidado & Sin estudios & 41,68 & 5,69 & 6,78 & $<0,001$ \\
& Primaria & 44,42 & 5,89 & & \\
& Secundaria & 46,39 & 4,12 & & \\
Prevención de enfermedades & Bachillerato y más & 49,42 & 4,79 & & \\
& Sin estudios & 37,72 & 3,99 & 5,19 & \\
& Primaria & 39,4 & 4,27 & & \\
& Secundaria & 41,4 & 4,31 & & \\
Promoción de la salud & Bachillerato y más & 42,67 & 4,14 & & \\
& Sin estudios & 29,45 & 4,04 & & \\
& Primaria & 33,94 & 5,54 & & \\
Acceder/obtener & Secundaria & 34,88 & 5,68 & & \\
& Bachillerato y más & 38,82 & 6,12 & & \\
& Sin estudios & 28,77 & 3,99 & & \\
& Primaria & 30,45 & 4,82 & & \\
\end{tabular}




\begin{tabular}{|c|c|c|c|c|c|}
\hline \multirow[t]{4}{*}{ Entender/comprender } & Sin estudios & 27.88 & 3,8 & 7,32 & $<0,001$ \\
\hline & Primaria & 30,38 & 3,8 & & \\
\hline & Secundaria & 30,92 & 3,77 & & \\
\hline & Bachillerato y más & 33,54 & 2,87 & & \\
\hline \multirow[t]{4}{*}{ Procesar/juzgar } & Sin estudios & 27,69 & 3,57 & 8,21 & $<0,001$ \\
\hline & Primaria & 30,8 & 3,41 & & \\
\hline & Secundaria & 31,95 & 3,35 & & \\
\hline & Bachillerato y más & 32,67 & 3,98 & & \\
\hline \multirow[t]{4}{*}{ Aplicar/usar } & Sin estudios & 25,31 & 2,58 & 3,43 & 0,021 \\
\hline & Primaria & 26,58 & 3,76 & & \\
\hline & Secundaria & 27,44 & 3,66 & & \\
\hline & Bachillerato y más & 28,92 & 4,01 & & \\
\hline
\end{tabular}

Anova de un factor. Grupos: 1) Sin estudios, 2) Primaria, 3) Secundaria, 4) Bachillerato o más
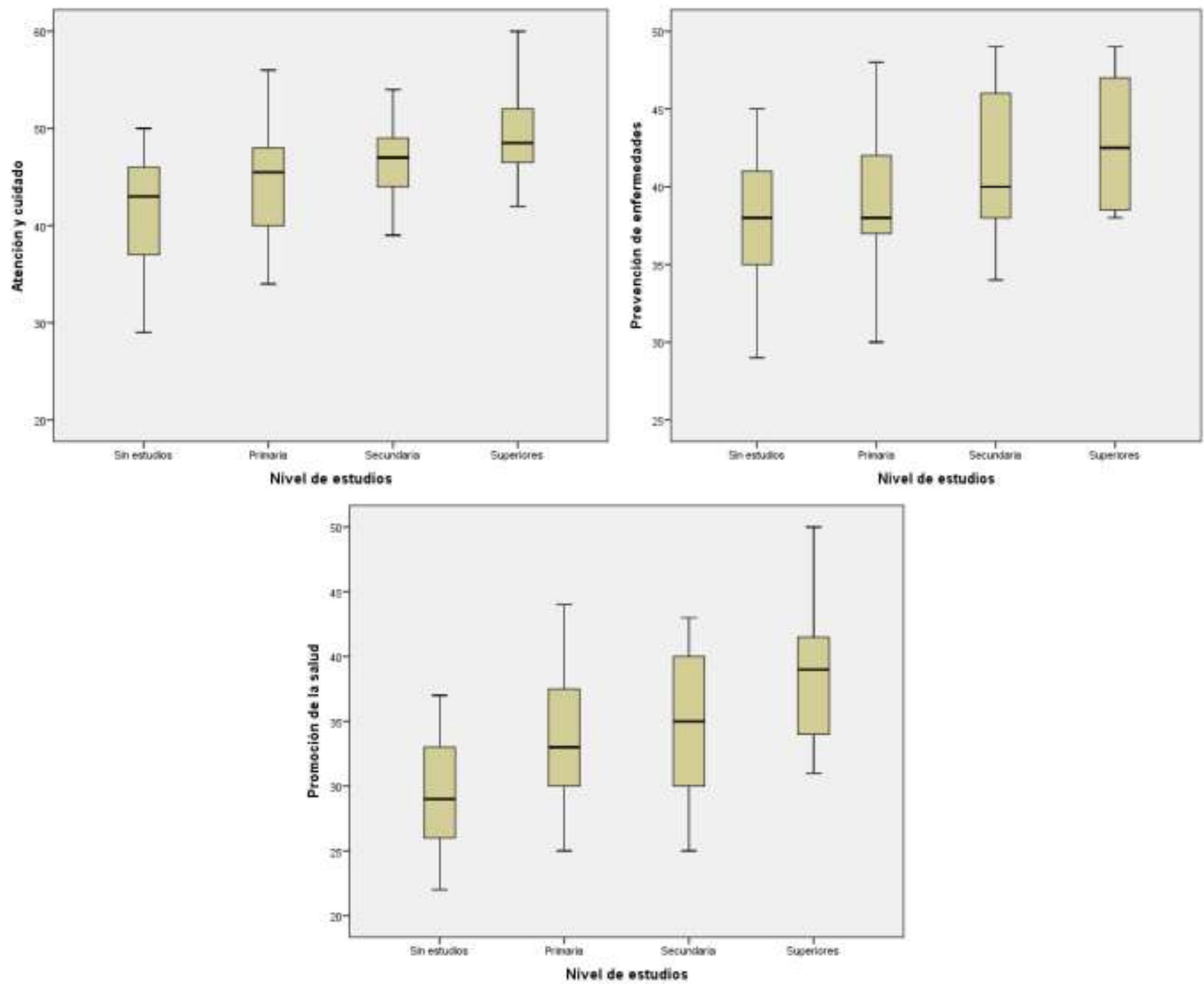

Figura 1. Medias de dimensiones de la alfabetización en salud por nivel de estudios

Mediante análisis de varianza se compararon las capacidades y dimensiones de AS en relación con los grupos de edad (<50 años, 51 a 65 años y 65 en adelante), encontrando diferencias significativas en la dimensión de promoción de la salud y la capacidad de acceder/obtener: a mayor edad, menor promoción de la salud y capacidad de acceder a la información de salud (Figura 2). 

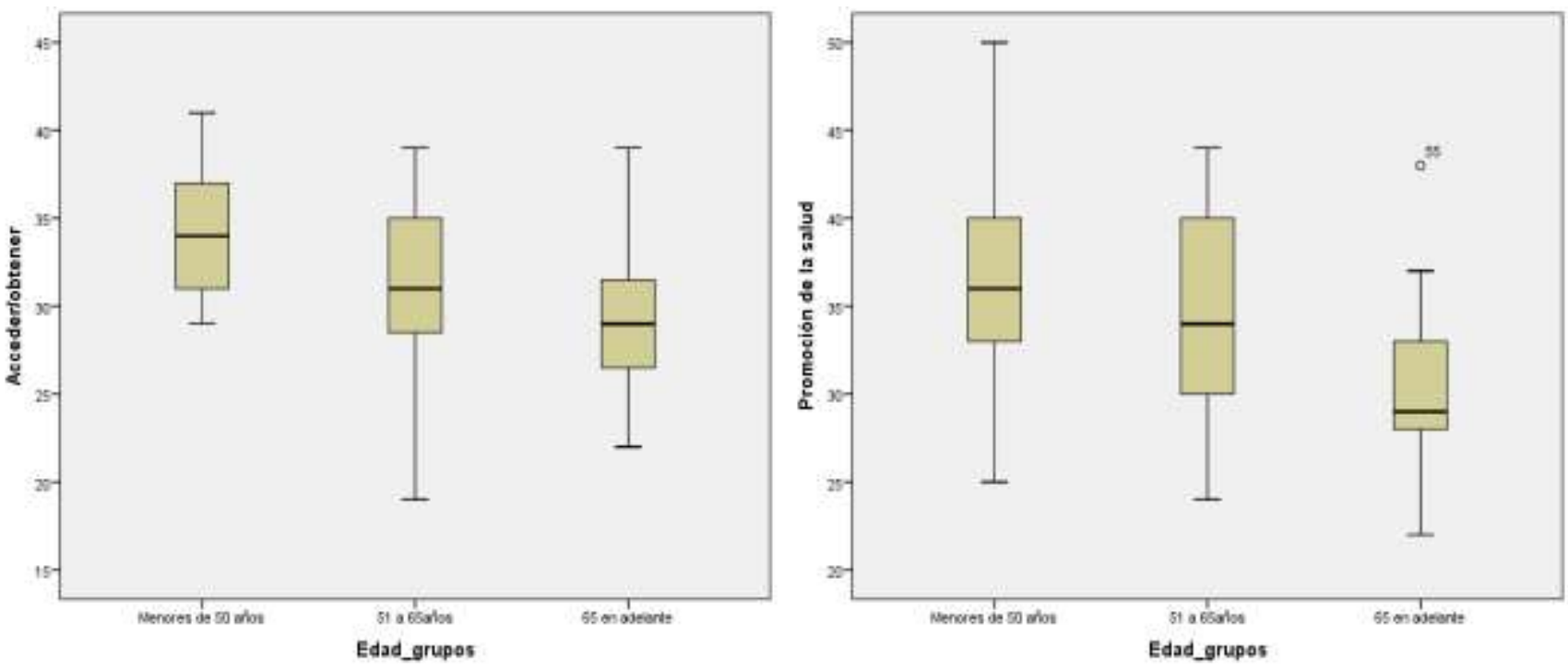

Figura 2. Medias de dimensiones de la alfabetización en salud por grupos de edad

El autocuidado de la dieta mostró correlación significativa con la AS en todas sus dimensiones y capacidades, a excepción de procesar/juzgar. De igual manera, correlacionó de forma inversa con la glucosa. El resto de las dimensiones de autocuidado no mostraron correlaciones significativas con la alfabetización en salud. La edad, correlacionó inversamente con las dimensiones de AS: prevención de enfermedades y promoción de la salud y las capacidades: acceder/obtener y procesar/juzgar (Tabla 4).

Tabla 4. Correlaciones entre nivel de alfabetización en salud, glucemia, conductas de autocuidado y edad

\begin{tabular}{lrrrrrr}
\hline & Dieta & Ejercicio & Monitoreo glucosa & Cuidado de pies & Medicamentos & Edad \\
\hline Alfabetización en salud & & & & & & \\
Atención y cuidado & $0,280^{* *}$ & 0,057 & 0,193 & 0,188 & $-0,055$ & $-0,112$ \\
Prevención de enfermedades & $0,274^{*}$ & 0,207 & 0,068 & 0,076 & $-0,014$ & $-0,216^{*}$ \\
Promoción de la salud & $0,324^{* *}$ & $-0,077$ & 0,235 & 0,077 & 0,064 & $-0,306^{*}$ \\
Acceder/obtener & $0,345^{* *}$ & 0,055 & 0,194 & 0,116 & $-0,060$ & $-0,303^{*}$ \\
Entender/comprender & $0,294^{* *}$ & 0,062 & 0,203 & 0,160 & 0,010 & $-0,195$ \\
Procesar/juzgar & 0,185 & $-0,047$ & 0,054 & 0,033 & 0,000 & $-0,246^{*}$ \\
Aplicar/usar & $0,317^{* *}$ & 0,175 & 0,107 & 0,086 & $-0,013$ & $-0,085$ \\
Glucemia & $-0,331^{* *}$ & 0,008 & 0,05 & $-0,114$ & 0,024 & $-0,084$ \\
\hline
\end{tabular}

${ }^{* *} \mathrm{p} \leq 0,001{ }^{*} \mathrm{p} \leq 0,05$

Considerando que el autocuidado de la dieta se asoció de forma significativa con las dimensiones de la alfabetización en salud, se desarrolló un modelo de regresión lineal para identificar variables predictoras. El puntaje de la prueba de DurbinWatson indica que hay independencia de errores
$(2,078)$ y el factor de varianza inflada muestra que se cumple con el supuesto de no multicolinealidad (valores entre 1,109 y 1,621). Se encontró que la dimensión de promoción de la salud se asocia significativamente con el autocuidado de la dieta (Tabla 5). 
Tabla 5. Análisis multivariado para la determinación de variables predictoras del autocuidado de la dieta

\begin{tabular}{lrrrrr}
\hline \multicolumn{1}{c}{ Alfabetización en salud } & B & Stardard error & $\boldsymbol{\beta}$ & t & p \\
\hline Constante & $-6,580$ & 7,74 & & $-0,850$ & 0,000 \\
Atención y cuidado & 0,168 & 0,16 & 0,18 & 1,04 & 0,303 \\
Prevención de enfermedades & $-0,212$ & 0,24 & $-0,17$ & $-0,88$ & 0,384 \\
Promoción de la salud & 0,417 & 0,17 & 0,43 & 2,44 & 0,018 \\
Edad & 0,132 & 0,07 & 0,25 & 2,00 & 0,051 \\
\hline
\end{tabular}

\section{Discusión}

El estudio de la AS y su relación con los resultados en salud, se ha realizado principalmente en individuos con enfermedades crónicas no transmisibles como hipertensión y diabetes, de igual forma se ha examinado su impacto en la adherencia a los medicamentos y su implicación social(11,15,21). Algunos estudios realizados en Estados Unidos de Norteamérica y Holanda, en pacientes con diabetes tipo 2 encuentran que la baja AS se asocia con la presencia de complicaciones y con el descontrol glucémico(22,23,16). Sin embargo, este enfoque se centra en una relación lineal entre AS y control glucémico, o AS y presencia de complicaciones, por lo que existe un vacío en el conocimiento con lo que respecta a los mecanismos que pudieran estar mediando en estas relaciones, tal es el caso de las conductas de autocuidado.

El 27,8\% de los participantes no contaba con estudios concluidos ( $<1$ año de primaria), situación que ha sido evidenciada en investigaciones como una limitante para el acceso a la información en salud y asociada a un bajo nivel de $\mathrm{AS}(14,24)$. Con este antecedente se hace evidente que la población de estudio presenta carencias para la lectura y la escritura, las cuales pudieran representar barreras para atender las recomendaciones de los profesionales de la salud toda vez que la lectura de las prescripciones y recomendaciones se ve limitada. Esto se ratifica en el hallazgo que muestra que el nivel de AS fue inadecuado o problemático para la mayoría de los participantes en el estudio.

En cuanto a las complicaciones derivadas de la diabetes referidas por los participantes, la más frecuente es retinopatía (36,7\%), en otro estudio al comparar pacientes con AS alta con quienes presentaban AS baja, los últimos tuvieron 2 veces mayor riesgo de tener retinopatía(22). En este sentido, las recomendaciones que resaltan la importancia de que el paciente con diabetes adopte y mejore las conductas de autocuidado para minimizar complicaciones a largo plazo se vuelven prioritarias(25). Esta alta frecuencia de retinopatía merece ser explorada a profundidad en estudios posteriores que permitan esclarecer si hay una relación causal.

En el autocuidado de la dieta poco más de la mitad $(53,4 \%)$ de los participantes refirieron seguir un plan de alimentación saludable de 3 a 5 días durante los últimos 7 días; estudios previos habían puesto en evidencia que las actividades de autocuidado de la dieta eran llevadas en promedio durante 4 días a la semana, también señalan que el autocuidado de la dieta es una de las áreas en donde se debe intervenir debido a su relación con el control glucémico y a la poca adherencia reportada por los individuos(13,26). En este sentido debe tenerse en consideración que para una persona que vive con diabetes el cuidado de la alimentación 4 días y descuido otros 3, puede representar altos riesgos a la salud por las fluctuaciones en los niveles de glucosa y con ello posibles complicaciones.

Por otra parte, el autocuidado de la dieta mostró correlación significativa con la AS en todas sus dimensiones y capacidades con excepción de la dimensión procesar/juzgar. Esto concuerda con lo reportado por Santesmases, et al., quienes encontraron que las pobres conductas de autocuidado se relacionan con el bajo nivel de comprensión de la información en salud(27). Si bien en la presente investigación, dado su diseño transversal, no es posible establecer causalidad, si apunta a una relación que pudiera ser sumamente útil en términos de prevención por lo que merece su estudio en investigaciones longitudinales.

Asimismo, el autocuidado en la dieta correlacionó de forma inversa con el nivel de glucosa en sangre, por lo cual ante mayor cuidado en la dieta menor nivel de glucemia en los pacientes. Al respecto Lee, et al., reportan en su estudio en Taiwán, que las actividades 
de autocuidado, en especial las de dieta y ejercicio, influyen en el control glucémico de los pacientes con diabetes tipo $2^{(28)}$.

La AS puede ser un componente clave para el autocuidado de los pacientes. La correlación encontrada entre AS y edad, sugiere que las personas mayores podrían enfrentar más dificultades en acceder, comprender y juzgar la información en salud. Es por ello que se debe plantear nuevas estrategias educativas que faciliten el proceso de comprensión de dicha información y tener presente que a medida que la edad avanza, el acompañamiento tanto de los profesionales de la salud como de las redes de apoyo social requiere ser más cercano para facilitar que sigan las recomendaciones de los profesionales de la salud. En algunos estudios se ha señalado que el crear intervenciones utilizando formatos como dibujos e imágenes podrían ayudar a comprender mejor los temas de promoción de la salud y con ello mejorar la $\mathrm{AS}(23,27)$.

\section{Conclusión}

El nivel de AS en pacientes con diabetes tipo 2 fue en su mayoría inadecuado, considerando que deben desarrollar múltiples actividades en el cuidado y control de su enfermedad (automonitoreo de la glucemia, toma de medicamentos, agenda de citas médicas, control de su dieta, etc.). Es indispensable fomentar estrategias de alfabetización en salud en especial cuando existe bajo nivel educativo y mayor edad. Esto se ratifica y pone en relieve al considerar la asociación identificada entre el autocuidado de la dieta y la AS, en específico en la dimensión de promoción de la salud; poniendo en evidencia la responsabilidad sobre AS que tienen los pacientes para su autocuidado.

\section{Agradecimientos}

Al Consejo Nacional de Ciencia y Tecnología de México, por brindar las facilidades para llevar a cabo la investigación, con la asignación de la beca de estudios de posgrado y de doble titulación al CVU 710504.

\section{Conflicto de intereses}

Los autores declaran de no existen conflictos de interés.

\section{Referencias}

1. Romero MF, Ruiz-Cabello AL. Alfabetización en salud; concepto y dimensiones. Proyecto europeo de alfabetización en salud. Rev Comun y Salud. 2012;2(2):91-98.

2. Nutbeam D. Health literacy as a public health goal: a challenge for contemporary health education and communication strategies into the 21st century. Heal Promot Int. 2000;15(3):259-267.

3. Gutiérrez JP, Rivera-Dommarco J, Shamah-Levy T, llalpandoHernández S, Franco A, Cuevas-Nasu L, et al. Encuesta nacional de salud y nutrición 2012. Resultados Nacionales. Cuernavaca, México: Instituto Nacional de Salud Pública; 2012.

4. International Diabetes Federation. IDF Diabetes Atlas. 7th ed. Brussels, Belgium: Int Diabetes Fed; 2015. [Citado 01 May 2017]; Disponible en: http://www.diabetesatlas.org/

5. Instituto Nacional de Salud Pública. Encuesta Nacional de Salud y Nutrición de Medio Camino 2016. Informe Final de Resultados [Internet]. México: INSP; 2016 [Citado 25 Abr 2017]. Disponible en: http://www.promocion.salud.gob.mx/dgps/descargas1/do ctos_2016/ensanut_mc_2016-310oct.pdf

6. Centro Nacional de Excelencia Tecnológica en Salud. Tratamiento de la diabetes mellitus tipo 2 en el primer nivel de atención [Internet]. México: CENETEC; 2014. [Citado 20 Abr 2017] Disponible en: http://www.cenetec.salud.gob.mx/descargas/gpc/Catalogo Maestro/718_GPC_Tratamiento_de_diabetes_mellitus_tipo_2 _/718GER.pdf

7. Olivella-Fernández MC, Bastidas-Sánchez CV, CastiblancoAmaya MA. La adherencia al autocuidado en personas con enfermedad cardiovascular: abordaje desde el modelo de Orem. Aquichan. 2012;12(1):53-61.

8. Secretaria de Salud, México. Norma Oficial Mexicana NOM015-SSA2-2010, Para la prevención, tratamiento y control de la diabetes mellitus. México: Secretaria de Salud; 2010.

9. Manzini FC, Simonetti JP. Nursing consultation applied to hypertensive clients: application of Orem's self-care theory. Rev Lat Am Enfermagem. 2009;17(1):113-119.

10. Trujillo-Olivera LE, Nazar-Beutelspacher A. Autocuidado de diabetes: una mirada con perspectiva de género. Estudios Demográficos y Urbanos. 2011;26(3):639-670.

11. Reisi M, Mostafavi F, Javadzade H, Mahaki B, Tavassoli E, Sharifirad G. Impact of health literacy, self-efficacy, and outcome expectations on adherence to self-care behaviors in iranians with type 2 diabetes. Oman Med J. 2016;31(1):5259.

12. Inoue $M$, Takahashi M, Kai I. Impact of communicative and critical health literacy on understanding of diabetes care and self-efficacy in diabetes management: a cross-sectional study of primary care in Japan. BMC Fam Pract. 2013;14(1):1-9.

13. Bohanny W, Wu SF, Liu CY, Yeh SH, Tsay SL, Wang TJ. Health literacy, self-efficacy, and self-care behaviors in patients with type 2 diabetes mellitus. J Am Assoc Nurse Pract. 2013;25(9):495-502.

14. Leung AYM, Cheung MKT, Chi I. Relationship among patients' perceived capacity for communication, health literacy, and diabetes self-care. J Health Commun. 2014;19(sup 2):161172. 
15. Friis K, Vind BD, Simmons RK, Maindal HT. The relationship between health literacy and health behaviour in people with diabetes: A Danish population-based study. J Diabetes Res. 2016;2016:1-7.

16. van der Heide I, Uiters E, Rademakers J, Struijs JN, Schuit AJ, Baan CA. Associations among health literacy, diabetes knowledge, and self-management behavior in adults with diabetes: results of a dutch cross-sectional study. J Health Commun. 2014;19(sup2):115-131.

17. Herrera-Lían A, Andrade Y, Hernández O, Manrique J, Faria K, Machado M. Personas con diabetes mellitus tipo 2 y su capacidad de agencia de autocuidado, Cartagena. Av en Enfermería. 2012;30:39-46.

18. Sørensen K, Pelikan JM, Röthlin F, et al. Health literacy in Europe: comparative results of the European health literacy survey (HLS-EU). Eur J Public Health. 2015;25(6):1053-1058.

19. Luna Ruiz-Cabello A. La alfabetización en salud de la población española: Variables relacionadas según los resultados del proyecto europeo de alfabetización en salud. Murcia: Universidad de Murcia; 2015.

20. Vincent D, McEwen MM, Pasvogel A. The validity and reliability of a Spanish version of the summary of diabetes self-care activities questionnaire. Nurs Res. 2008;57(2):101106.

21. Fan JH, Lyons SA, Goodman MS, Blanchard MS, Kaphingst KA. Relationship Between Health Literacy and Unintentional and Intentional Medication Nonadherence in Medically Underserved Patients With Type 2 Diabetes. Diabetes Educ. 2016;42(2):199-208.
22. Schillinger D, Grumbach K, Piette J, Wang F, Osmond D, Daher $\mathrm{C}$, et al. Association of health literacy with diabetes outcomes. JAMA. 2002;288(4):475-82.

23. Hersh L, Salzman B, Snyderman D. Health Literacy in Primary Care Practice. Am Fam Physician. 2015;92(2):118-24.

24. Friis K, Lasgaard M, Rowlands G, Osborne RH, Maindal HT. Health literacy mediates the relationship between educational attainment and health behavior: A Danish population-based study. J Health Commun. 2016;21(sup 2):54-60.

25. Gamboa-Moreno E, Ochoa de Retana-García L, del CampoPena ME, Sánchez-Perez A, Catalina Martínez-Carazo C, Arbonies-Ortiz JC, et al. A Pilot Study to Assess the Feasibility of the Spanish Diabetes Self-Management Program in the Basque Country. J Diabetes Res. 2016;2016:1-11.

26. Eyüboğlu E, Schulz PJ. Do health literacy and patient empowerment affect self-care behaviour? A survey study among Turkish patients with diabetes. BMJ Open. 2016;6(3):e010186.

27. Santesmases-Masana R, González-de Paz L, Real J, BorràsSantos A, Sisó-Almirall A, Navarro-Rubio MD. Alfabetización en salud en pacientes con insuficiencia cardiaca atendidos en atención primaria. Atención Primaria. 2017;49(1):28-34.

28. Lee EH, Lee YW, Moon SH. A Structural Equation Model Linking Health Literacy to Self-efficacy, Self-care Activities, and Health-related Quality of Life in Patients with Type 2 Diabetes. Asian Nurs Res (Korean Soc Nurs Sci). 2016;10(1):82-87. 